\title{
The Covid-19 Pandemic and its Effect on the Elderly: A Review
}

\author{
Maimoona Sajid Nadri ${ }^{1 *}$ \\ ${ }^{1}$ Jinnah Postgraduate Medical Center, Pakistan \\ *Corresponding author: Maimoona Sajid Nadri: maimoonanadri@gmail.com
}

\section{OPEN ACCESS}

Citation: Nadri M.S. (2020) The Covid Pandemic and its Effect on the Elderly: A Review. Open

Science Journal 5(3)

Received: $1^{\text {st }}$ July 2020

Accepted: $31^{\text {st }}$ August 2020

Published: $2^{\text {nd }}$ October 2020

Copyright: (c) 2020 This is an open access article under the terms of the Creative Commons Attribution License, which permits unrestricted use, distribution, and reproduction in any medium, provided the original author and source are credited.

Funding: The author received no specific funding for this work

Competing Interests: The author has declared that no competing interests exist.

\section{Abstract:}

In 2019, the world experienced a global public health concern when the novel coronavirus originated from China and affected around 22 million people around the world by August 2020. The quick rise in the number of cases, and the death toll overwhelmed the scientific and medical community. While all the focus was driven towards finding the epidemiology, the treatment, and the management, the mental health aspect of the quarantine due to COVID-19 was being overlooked. The elderly are a vulnerable population and prone to physiological and psychological aftermath of the pandemic. Recognizing this issue, the purpose of this review is to create awareness about the negative consequences (outcomes) of quarantine, focusing on the elderly community in Pakistan.

Keywords: Covid-19, Elderly, Mental health

\section{Introduction}

The world experienced a global public health concern when the Coronavirus pandemic hit, claiming approximately 800,000 lives and infecting 22,000,000 people around the world as of August 2020 [1]. Covid-19 is an infectious disease caused by a novel coronavirus named SARS-CoV-2 (severe acute respiratory syndrome- coronavirus). Coronaviruses are known to have caused other respiratory infections including Middle East Respiratory Syndrome (MERS-CoV) and Severe Acute Respiratory Syndrome (SARS-CoV).

The symptoms of COVID-19 appear after an incubation period of 5.2 days and they range from fever, cough, fatigue, and diarrhea to life threatening complications like acute respiratory distress syndrome (ARDS), acute cardiac dysfunction and death. Adults greater than sixty years old with reduced immunity had a shorter incubation period and a higher risk of severity of the 
disease. Elderly with comorbidities like hypertension, diabetes and cardio vascular diseases had a higher rate of Intensive Care Unit (ICU) admissions [2-4].

Despite the observation of increased vulnerability in the elderly scant data is present regarding the effects of COVID-19 in the elderly.

Almost one third of the world was under lockdown from March to April and Pakistan was amongst one of the countries to implement it strictly. In order to limit the spread of infection, strict measures were executed to contain it which involved stay-at-home orders, implementation of handwashing techniques or using alcohol- based hand sanitizers, mandatory covering of nose and mouth in public and limiting social contact [5]. Social distancing was practiced almost everywhere, and all non-essential activities were suspended. People, especially the elderly being more vulnerable were made to quarantine themselves, limiting their social contact and disturbing their daily schedules resulting in mental dismay. Due to the special measures that quarantine brings they were not only suffering from the physiological, but the psychological impact of the disease being prone to isolation, stress, depression and anxiety during the quarantine [6].

The literature search for this review was carried out on PubMed and Google Scholar using keyword "COVID-19", "coronavirus" and "pandemic", crossreferencing it with "mental health", "elderly", "depression" and narrowed down our search to the articles which were written in the English language and their primary focus was the elderly population. This review focuses on the effects of quarantine in the elderly during the pandemic and its long-term consequences on their mental health and assesses the measures required to prevent temporary or permanent negative outcomes.

\section{Discussion}

The world has recovered from massive infectious disease outbreaks like the Plague, Spanish flu, H1N1, Ebola, SARS and MERS. In those challenging times the focus was on epidemiology, treatment, containing the spread of the infection and to recover the ailing. Limited focus was given on the consequence it would have on the mental health of the people. Countries which lack proper health care systems and investments in the public health sector suffer the most.

An analysis of the previous epidemics gives us an insight on the outcomes it had on the mental health of the aging population.

\section{The effects of social isolation in the past}

The MERS-CoV and SARS-CoV epidemic in 2012 and 2003 respectively had a significant impact on the mental health of the society [7, 8]. Quarantine and isolation were one of the main non-pharmacological methods used to control the disease, however, after the epidemic was over long-term effects like posttraumatic stress disorder (PTSD), anxiety and depression were observed even after a year in the affected population [9]. The most vulnerable of them were the elderly from the ages of 60 and above, for whom social contact and interaction were essential to protect them from loneliness, depression and severe consequences for example suicide [10]. 
The effects of quarantine in the COVID-19 pandemic and effective required measures

Most of the data available online regarding the COVID-19 focuses on the general population, with limited focus given solely to the geriatric population. One general population study done in Pakistan showed that $83.8 \%$ of the participants felt anxious, and $86.5 \%$ limited physical contact with people. The participants were also noted to avoid activities that made them anxious about the situation and adapted practices in their routines to protect themselves from getting infected. It included avoiding going to the markets, work, and places of worship disrupting their daily routines [11]. The elderly population (ages $>65$ ) were seen to be more susceptible to the disease and being more restricted forced them to withdraw themselves from the rest of the community, causing fear and stress amongst the seniors. Countries like China, The United Kingdom and The United States of America have analyzed the effects of quarantine and the studies show there are a large number of elderly population, both men and women, home-dwelling and nursing home residents at the risk of developing psychological sequels caused by the pandemic crisis [12-14].

Families play an important role in the Pakistani society; elderlies hold a place of respect and authority, with children being their future support. Family caregivers need to be vigilant and be educated by health care providers about any change in behavior or routine which may require a referral to a mental health care provider or facility for timely intervention. Those adults with preexisting mental health issues like dementia and Alzheimer's, depression and anxiety may be at a risk of relapse due to disruption of routine because of the quarantine or even absence of their regular care-givers, all elective procedures and regular checkups were also suspended to avoid unnecessary exposure causing a deterioration in their general health condition [6].

A general study conducted in Pakistan which focused on depression showed that elderly with poor social and financial support, illiteracy and lack of awareness suffered from deteriorating mental health which in times of the pandemic should make us concerned and give us guidance on how to construct effective strategies to cope with the mental health problems which we are going to encounter during and after the pandemic. [15, 16]

A strong association was found between depression and lack of physical activity and activities of daily living and even physiotherapy which has been considerably reduced because of the COVID-19 pandemic [18], for these reason caregivers should be encouraged to help maintain a regular physical activity schedule in accordance with the COVID-19 social distancing guidelines.

To reduce the long-lasting impact of pandemic, actions need to be taken now. Providing accurate and adequate information is important as unfamiliarity of the situation often causes fear and distrust amongst the masses. The purpose of quarantine must be explained to improve adherence that is critical in keeping the elderly especially with mental health issues, like dementia, anxiety, depression and comorbidities safe.

Even though a pandemic has been globally declared and still in effect, most of the countries including Pakistan have started to lift the stay-at-home order after taking precautionary measures to prevent another wave of COVID-19, limiting the exposure to the stressors affecting their psychological health. In the case of post-lockdown circumstances, society needs to be accountable for taking the necessary precautions as an instant eradication of danger is impossible.

With the advancement of technology, we need to learn how to use it to our benefit and make platforms available allowing experts to answer queries and 
increase knowledge whilst making it accessible to the population in need. Increasing ways of communication through telephone and internet via video conferencing and other means of communication can make the elderly feel integrated to the world and reduce the boredom and sense of loneliness that comes with social isolation and making them feel connected emotionally if not physically in these tough times [5].

Telehealth has gained popularity again as hospital visits are best avoided during these times, keeping mental health in mind steps have been taken by the Sindh government in Pakistan by launching a hotline for mental health in collaboration with UNICEF and UNDP in response to the pandemic [17].

It is important that we do not halt any of the ongoing research because of the pandemic, rather, take advantage of this time and make advancements in the areas such as geriatrics which lack significant analysis. Nicol et al. in their report has guided future researchers on how to safely continue with their researches involving the geriatric population. This involves keeping remote contact by utilizing digital tools to keep the participants engaged, informed, and equip the research staff with proper resources [19].

\section{Conclusion}

These are unprecedented times; the medical and scientific communities need to adapt to the changes caused by the pandemic which might become the new norm. The twenty-first century has already experienced consequential infectious disease outbreaks which have changed the outlook of medical sciences. Analyzing prior studies have pointed out that this pandemic will have a long-lasting effect on the psychological well-being of the susceptible community. The elderly's physical and psychological needs are to be given special attention. If appropriate measures are taken on time, we can reduce the burden on the health care system.

\section{References:}

1.WHO. Coronavirus disease (COVID-19) situation report- 209. 2020.

2.Li Q, Guan X, Wu P, Wang X, Zhou L, Tong Y, et al. Early Transmission Dynamics in Wuhan, China, of Novel Coronavirus-Infected Pneumonia. N Engl J Med. 2020;382(13):1199-207.

3.Rothan HA, Byrareddy SN. The epidemiology and pathogenesis of coronavirus disease (COVID-19) outbreak. J Autoimmun. 2020;109:102433.

4.Wang D, Hu B, Hu C, Zhu F, Liu X, Zhang J, et al. Clinical Characteristics of 138 Hospitalized Patients With 2019 Novel Coronavirus-Infected Pneumonia in Wuhan, China. JAMA. 2020;323(11):1061-9.

5.WHO. World Health Organization Corona Virud Disease (COVID-19) Advice for the public. April, 2020.

6.Banerjee D, D'Cruz M, Sathyanarayana Rao TS. Coronavirus disease 2019 and the elderly: Focus on psychosocial well-being, agism, and abuse prevention - An advocacy review. Journal of Geriatric Mental Health. 2020;7(1).

7.N.S. AlNajjar LMA, F.M. Farahat, A.AlThaqafi. Psychobehavorial responses to the 2014 Middle East respiratory syndrome-novel corona virus (MERS CoV) among adults in two shopping malls in Jeddah, western Saudia Arabia. Eastern Mediterranian Health Journal 2016;22.

8.Lau AL, Chi I, Cummins RA, Lee TM, Chou KL, Chung LW. The SARS (Severe Acute Respiratory Syndrome) pandemic in Hong Kong: effects on the subjective wellbeing of elderly and younger people. Aging Ment Health. 2008;12(6):746-60.

9.Wu KK, Chan SK, Ma TM. Posttraumatic stress, anxiety, and depression in survivors of severe acute respiratory syndrome (SARS). J Trauma Stress. 2005;18(1):39-42. 
10.Yip PS, Cheung YT, Chau PH, Law YW. The impact of epidemic outbreak: the case of severe acute respiratory syndrome (SARS) and suicide among older adults in Hong Kong. Crisis. 2010;31(2):86-92.

11.Balkhi F, Nasir A, Zehra A, Riaz R. Psychological and Behavioral Response to the Coronavirus (COVID-19) Pandemic. Cureus. 2020;12(5):e7923.

12.Meng H, Xu Y, Dai J, Zhang Y, Liu B, Yang H. Analyze the psychological impact of COVID-19 among the elderly population in China and make corresponding suggestions. Psychiatry Res. 2020;289:112983.

13.Morrow-Howell N, Galucia N, Swinford E. Recovering from the COVID-19 Pandemic: A Focus on Older Adults. J Aging Soc Policy. 2020;32(4-5):526-35.

14.N R. Risks to the elderly during the coronavirus (COVID-19) pandemic 2019-2020. Journal of Geriatric Care and Research 2020.

15.Bhamani MK, M;Khan, M. Depression in the elderly in Karachi, Pakistan: a cross sectional study. BMC Psychiatry. 2013.

16.S.M. Mubeen DH, S. N. Qureshi. Prevalence of Depression Among Community Dwelling Elderly in Karachi, Pakistan. Iran J Psychiatry Behav Sci. 2012;6.

17.Samaa(2020). Sindh launches corona virus mental health support hotline. 2020. 